FORMATION Formation emploi

Revue française de sciences sociales

150 | avril-juin 2020

Former aux "petits" métiers : regards internationaux

\title{
La SEGPA : des socialisations professionnelles précoces au collège
}

SEGPA : early professional socialisation in secondary school

SEGPA : Die frühzeitige berufliche Sozialisation in der Sekundarschule

SEGPA : socializaciones profesionales precoces en el primer ciclo de la enseñanza secundaria

\section{Charlotte Moquet}

\section{OpenEdition}

\section{Journals}

Édition électronique

URL : https://journals.openedition.org/formationemploi/8124

DOI : 10.4000/formationemploi.8124

ISSN : 2107-0946

Éditeur

La Documentation française

Édition imprimée

Date de publication : 8 juillet 2020

Pagination : 55-78

ISSN : 0759-6340

Référence électronique

Charlotte Moquet, "La SEGPA : des socialisations professionnelles précoces au collège », Formation emploi [En ligne], 150 | avril-juin 2020, mis en ligne le 02 janvier 2022, consulté le 07 janvier 2022. URL : http://journals.openedition.org/formationemploi/8124 ; DOI : https://doi.org/10.4000/ formationemploi.8124 


\title{
La SEGPA : des socialisations professionnelles précoces au collège
}

\author{
Charlotte Moquet \\ Doctorante en sociologie au sein de l'École doctorale Sciences de la société, territoires, sciences \\ économiques et de gestion (SSTSEG) de l'université de Poitiers et du Laboratoire Groupe de \\ recherches sociologiques sur les sociétés contemporaines (GRESCO)
}

Résumé

La SEGPA : des socialisations professionnelles précoces au collège

La section d'enseignement général et professionnel adapté (SEGPA) se situe en amont des scolarités et formations professionnelles en lycée ou en centre de formation d'apprentis. Elle comporte des enseignements professionnels, ateliers et stages, qui ont lieu durant la 4e et la 3e. Malgré son inscription en collège et les représentations qui lui sont associées, la SEGPA est bien professionnelle au sens où elle soumet déjà des collégiens à des socialisations professionnelles. Elle est le lieu d'une fabrication de futurs élèves de "petits » CAP (certificat d'aptitude professionnelle) en lycée (polyvalents et peu sélectifs) et de dispositions professionnelles renvoyant à des postes subalternes, d'exécution et polyvalents.

Mots clés : collège, socialisation professionnelle, enseignement technique-professionnel, orientation scolaire-professionnelle, CAP - certificat d'aptitude professionnelle, contenu de formation

Abstract

SEGPA: early professional socialisation in secondary schools

The section of adapted general and vocational education (SEGPA), in France, takes place before schooling and vocational training in high schools or apprentice training centres. It includes vocational training, workshops and internships, for 14 to 16 years old students. Although it is in secondary school and despite its representations, SEGPA is indeed vocational in the sense that it already subjects secondary school students to professional socialisation. It is the place to produce future students with «small " vocational qualification (CAP is level 1 in France) and with working dispositions referring to subordinate, implementation and polyvalent jobs.

Keywords: lower secondary school, professional socialisation, technical \& vocational education, school guidance $\&$ vocational guidance, certificate of vocational aptitude, training content

Journal of Economic Literature : I 21

Traduction : auteure. 


\section{Introduction}

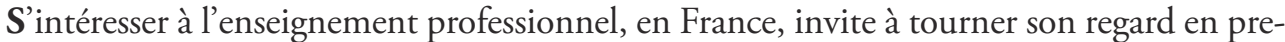
mier lieu vers les voies professionnelles du second cycle du secondaire que sont les lycées professionnels (LP) ou les centres de formation d'apprentis (CFA). Les sociologues ont ainsi étudié ces deux voies de formation (Moreau, 2008a ; Palheta, 2012), les diplômes qui y sont délivrés (Maillard, 2013 ; Orange, 2013) et certaines filières et métiers auxquels elles préparent (Denave \& Renard, 2015). A contrario, peu nombreuses sont les recherches sur les scolarités qui précèdent (premier cycle du secondaire) et qui intègrent, dans leurs contenus, des enseignements professionnels, "pré-professionnels " ${ }^{1}$ ou de "découverte professionnelle ", telles que la section d'enseignement général et professionnel adapté (SEGPA). L'article de Joël Zaffran (2010) est l'un des rares écrits sociologiques qui porte spécifiquement sur la SEGPA. L'auteur y propose une analyse quantitative des inégalités sociales à l'entrée et à la sortie de la section, à l'aide des données du Panel 1995 de la DEPP. Cependant, ce matériau ne permet pas de saisir ce qui se passe précisément durant ces années de scolarité et donc les socialisations qui y sont à l'œuvre.

\section{Encadré 1. La section d'enseignement général et professionnel adapté (SEGPA)}

La SEGPA porte ce nom depuis 1996 et a remplacé progressivement la section d'éducation spécialisée (SES, créée en 1967) à partir de 1989. La SEGPA est avant tout présentée comme une filière adaptée à des élèves dont le parcours à l'école primaire est marqué par des difficultés dans les apprentissages ${ }^{(1)}$. Présente au sein de certains collèges, elle est constituée des quatre niveaux du collège, de la $6^{\mathrm{e}}$ à la $3^{\mathrm{e}}$. Les enseignements y sont à la fois " généraux 》 (mais dits « adaptés ») et " professionnels » en $4^{\mathrm{e}}$ et en $3^{\mathrm{e}}$ du fait de la réalisation de stages en entreprise et du suivi d'ateliers. Pour chaque SEGPA, deux ateliers professionnels, dirigés par des professeurs des lycées professionnels (PLP), sont proposés aux élèves parmi cinq domaines: " hygiène, alimentation, services » (HAS), « habitat », " production industrielle », « espace rural et environnement » (ERE) et « vente, distribution, magasinage ». Les stages se font en 4e (deux stages d'une semaine) et en $3^{e}$ (deux à trois stages de deux semaines). Les élèves changent d'entreprise, voire de secteur professionnel, pour chacun d'eux.

De la $6^{e}$ à la $3^{e}$, les enseignements généraux sont principalement assurés par des professeurs des écoles (PE) spécialisés. II peut arriver que certains professeurs des lycées et collèges (PLC) y interviennent, notamment pour des enseignements tels que l'Éducation physique et sportive (EPS), la musique, les arts plastiques et l'anglais. Pour chaque SEGPA, un poste de directeuradjoint est créé au sein de l'équipe de direction du collège, qui coordonne et anime le travail de l'équipe enseignante de SEGPA, participe au suivi des élèves, etc.

1. Les citations entre guillemets, en italique et sans référencement sont issues du terrain (observations, entretiens, documents pédagogiques et institutionnels). 
L'entrée en SEGPA fait suite à la constitution d'un dossier, qui réunit des bilans scolaires, psychologiques, sociaux et médicaux, et à son étude par la Commission départementale d'orientation (CDO). Enfin, à l'issue de la 3e, les élèves passent majoritairement le Certificat de formation générale (CFG) qui est un examen scolaire ${ }^{(2)}$. Pour autant, et en lien avec l'objectif de diplomation de toute une classe d'âge depuis les années 1980, le but de la SEGPA est d'amener « tous les élèves [... ], à l'issue de la scolarité obligatoire [16 ans], [...] à une formation conduisant au minimum à une qualification de niveau $V »^{(3)}$, aujourd'hui « niveau $3 »^{(4)}$, soit un Certificat d'aptitude professionnelle (CAP).

En 2016, les élèves scolarisés en SEGPA représentent 2,6 \% des effectifs scolarisés en collège (soit 86300 élèves) ${ }^{(5)}$. Principalement des garçons (62 \% des effectifs), ils sont majoritairement " issus d'un milieu social défavorisé »(6).

(1) : De fait, " les élèves de Segpa ont presque tous un an de retard par rapport à l'âge théorique: seulement $8,5 \%$ sont "à l'heure", contre $87,4 \%$ des élèves de collège hors SEGPA », (MEN, DEPP-RERS, 2017, Repères et Références Statistiques sur les enseignants, la formation et la recherche, p. 120).

(2) : La DEPP ne connaît pas les résultats à cet examen. En revanche, très peu d'élèves passent et obtiennent le Diplôme national du brevet (DNB). Cf. MEN, DEPP, Note d'information $n^{\circ} 02$, janvier 2017, p. 2.

(3) : Circulaire n²015-176 du 28 octobre 2015, « sections d'enseignement général et professionnel adapté ».

(4) : Site Service-public.fr, "Quelle est la nomenclature des diplômes par niveau ? », consulté en juin 2020.

(5) : MEN, DEPP, Géographie de l'école, n 12, avril 2017, p. 26.

(6) : MEN, DEPP, Note d'information $n^{\circ} 02$, op. cit.

Statistiquement, les élèves de SEGPA poursuivent leur formation après le collège, le plus souvent dans des voies professionnelles ${ }^{2}$ (Zaffran, op. cit. ; Palheta, 2011). De ce fait, la SEGPA est une "antichambre de l'enseignement professionnel ", à l'instar de l'ensemble des «classes atypiques» au collège (Palheta, 2011). Pour autant, elle revêt certaines spécificités qui justifient de la saisir de manière autonome et qui la différencient des autres scolarités "spécialisées etlou pré-professionnelles" (ibid.). Les élèves y entrent, pour la majorité d'entre eux, dès l'entrée au collège en $\sigma^{\mathrm{e}}$ et n'en sortent qu'à la fin de la classe de $3^{\mathrm{e}}$ (Zaffran, op. cit.). En ce sens, elle soumet les élèves, au sein du collège, à un programme socialisateur sur une plus longue durée que les autres classes ou dispositifs alternatifs au collège général (par exemple, la $3^{\mathrm{e}}$ " prépa-métier $»^{3}$ ne dure qu’un an et est souvent intégrée au sein des lycées professionnels).

2. «L'orientation des élèves après la Segpa est une orientation majoritairement tournée vers la voie professionnelle, et principalement le CAP» (DEPP-RERS, op. cit., p. 120).

3. Anciennement appelée 3e "prépa-pro » qui a elle-même remplacé la « DP6 » (Découverte professionnelle six heures). 
Les contenus des enseignements dits professionnels renvoient à ceux dispensés dans la voie professionnelle, opposée et différenciée des voies générale et technique dans le système scolaire français. Ces enseignements s'inscrivent dans une perspective d'accès à des métiers et emplois catégorisés comme "manuels ", en opposition à des métiers " intellectuels ». Cette catégorisation indique davantage la position sociale du métier que la nature des tâches ou des activités qui y sont liées (Grignon, 1972). Ces enseignements, enfin, "mettent au travail » les élèves, c'est-à-dire les immergent dans des situations proches du monde du travail et ce, plus précocement dans la scolarité que pour les autres élèves, puisqu'ils effectuent leurs premiers stages dès la $4^{\mathrm{e}}$.

Les éléments qui précèdent invitent à appréhender la SEGPA sous l'angle des socialisations professionnelles. Mais plusieurs précisions sont alors nécessaires. Les résultats présentés ici n'ambitionnent pas de saisir des socialisations professionnelles « en acte et en situation", liées à l'occupation d'un poste de travail (Zolesio, 2012 ; Lahire, 1993). L'étude ne porte pas sur les processus, les instances ou les produits des socialisations en situation d'emploi : l'appréhension des socialisations professionnelles se fait sur le temps de la formation, en école (Darmon, 2015 ; Bertrand, 2012), à partir des enseignements professionnels qui y sont dispensés. En ce sens, ce sont les socialisations professionnelles « initiales» qui font l'objet de ce travail (Avril, Cartier \& Serre, 2010).

L'analyse porte plus précisément sur le cadre ou le programme socialisateur. L'accent est ainsi mis sur ce à quoi sont soumis les élèves durant leur scolarité en SEGPA, à quoi elle les forme, à quoi elle les destine, en laissant de côté, le temps de cet article, les questions de transmissions effectives de dispositions professionnelles chez les élèves. L'enjeu est de comprendre la socialisation " en train de se faire » en SEGPA (Darmon, $o p$. cit.) et d'interroger le caractère professionnel de certains de ses produits en s'arrêtant à l'identification des savoirs, techniques et rapport au travail auxquels sont confrontés les élèves le temps de cette formation.

Il s'agit donc, en prenant appui sur une enquête de terrain (cf. encadré 2), d'interroger les dispositions professionnelles que les élèves de SEGPA sont amenés à acquérir alors même qu'ils sont encore au collège, au travers notamment des discours et représentations des professeurs des "champs professionnels", de leurs pratiques en ateliers et autour des stages. Si des variations s'observent entre établissements, entre enseignants de SEGPA, elles ne sont ici pas l'objet de l'analyse. Tout l'enjeu est de saisir la SEGPA en tant qu'instance de socialisation professionnelle à part entière.

L'article propose alors d'éclairer le type d'élèves et de travailleurs que le cadre socialisateur de la SEGPA entend façonner, en trois temps. Bien que la SEGPA ne soit pas une formation professionnelle diplômante, son organisation et ses enseignements professionnels, ainsi que le travail institutionnel d'encadrement des aspirations des élèves sont tournés vers la voie professionnelle. Plus particulièrement, les élèves sont conduits vers des CAP en lycée, polyvalents et peu sélectifs (partie 1). Mais ces enseignements sont 
aussi marqués par un programme socialisateur qui apprend déjà aux élèves à devenir certains travailleurs. D'une part, les élèves sont soumis à un travail d'acculturation à des caractéristiques du travailleur d'exécution et subalterne (partie 2). D'autre part, la socialisation à l'œuvre n'est pas celle d'un métier mais bien plus d'emplois polyvalents. Elle renvoie davantage aux prémices d'une socialisation à un destin social, celui d'homme ou de femme «à tout faire » (partie 3).

\section{Encadré 2. Méthodologie}

L'article s'appuie sur une recherche menée à partir d'une enquête de type ethnographique, effectuée sur un temps long, entre 2012 et 2019, dans un département dont le pourcentage d'élèves scolarisés en SEGPA (section d'enseignement général et professionnel adapté) est proche de la moyenne nationale $(2,8 \%)^{(1)}$. Pour chacun des quatre collèges investigués, observations, entretiens, documents pédagogiques et institutionnels ont été produits en vue d'un croisement des matériaux. Quatre périodes de deux à trois semaines d'observations en continu ont été effectuées auprès d'élèves de $4^{e}$ et de $3^{e}$, non seulement des cours et ateliers, mais aussi des événements " hors classe » (conseils de classes, réunions d'équipe, rendez-vous parentsprofesseurs...). Par ailleurs, des entretiens ont été réalisés avec les différents agents des SEGPA (professeurs des lycées professionnels - PLP -, professeurs des écoles, directeurs), des élèves et parents d'élèves. Le travail d'orientation en SEGPA au niveau départemental a également été étudié par l'observation des séances de la Commission départementale d'orientation (CDO) sur trois années et des entretiens avec certains agents y siégeant.

Éclairés par l'ensemble de l'enquête, les matériaux les plus mobilisés sont ceux qui se rattachent aux enseignements professionnels de la SEGPA. II s'agit notamment des observations en atelier (huit séances dans cinq ateliers hygiène-alimentation-service, habitat ou espace rural environnement), de l'observation d'une vingtaine de visites de stages effectuées par les enseignants auprès des maîtres de stage, des observations des oraux blancs du Certificat de formation générale (CFG) qui portent sur la présentation d'un stage (quatre sessions dans deux SEGPA). Enfin, l'analyse s'appuie sur les entretiens effectués avec six PLP.

(1) : Si la moyenne nationale est de 2,6\%, les effectifs de SEGPA, par rapport à l'ensemble des collégiens, varient, selon les départements, entre 0,9\% (Paris) et 5,7 \% (Guyane). Cf. MEN, DEPP, Géographie de l'école, op. cit., fiche 8 « La scolarisation en collège » (site education.gouv.fr, consulté en janvier 2020).

\section{Des élèves orientés vers des « $p^{\prime}$ tits » CAP en lycée}

La SEGPA oriente ses élèves vers une poursuite de scolarité en formation professionnelle. En ce sens, elle est marquée par l'objectif de maintenir les élèves à l'école, mais aussi par l'injonction à l'obtention d'un diplôme. Les voies professionnelles de formation ne sont pas un espace homogène, mais hiérarchisé selon les filières, les diplômes, les spécialités, etc. (Palheta, 2012). Par suite, les injonctions enseignantes projettent 
les élèves dans un espace des possibles spécifique et délimité. Si le CAP est la référence, les élèves sont également invités à considérer certains CAP plutôt que d'autres et à privilégier le lycée au CFA ${ }^{4}$.

\subsection{Maintenir les élèves à l'école jusqu'au diplôme et les projeter dans le travail}

L'enseignement professionnel en SEGPA s'adjoint d'un discours de maintien des élèves à l'école, au moins jusqu'à l'obtention d'un diplôme, reflet des injonctions à limiter les sorties sans qualification du système scolaire, y compris pour des élèves jugés en difficulté.

Aux dires des PLP, l'objectif même de la SEGPA, dans son ensemble, est de « donner envie de venir " à l'école ; leur rôle premier est ainsi d' "accrocher » les élèves à travers leurs enseignements. Les élèves de SEGPA ne sont pas considérés comme "inscolarisables", contrairement aux élèves des dispositifs-relais ${ }^{5}$ (Millet \& Thin, 2003, p. 34), mais le retrait précoce de l'institution scolaire (avant 16 ans et avant l'obtention d'un diplôme) est perçu comme un risque à éviter. Les enseignements professionnels vont jusqu'à être qualifiés de "prétextes" par l'une des enseignantes pour faire acquérir les savoirs scolaires. S'exprime ici une fonction de remédiation scolaire qui rappelle les évolutions post-années-1980 de certaines filières professionnelles, notamment en CAP (Maillard, 1993).

La SEGPA n'est pas en elle-même sanctionnée par un diplôme professionnel. Ainsi, pour l'ensemble des agents en charge de l'orientation des élèves (conseillère d'orientation psychologue, directeur de SEGPA et enseignants), le but est de trouver à chaque élève de SEGPA une place dans une formation diplômante à la sortie du collège, et non pas de les préparer à entrer directement dans le monde du travail. Les élèves sont incités à privilégier toute opportunité permettant de suivre une formation, plutôt que de risquer de se retrouver «sans rien " après la $3^{\mathrm{e}}$ :

"Mais y'a la réalité, on essaie aussi d'leur faire comprendre que... bon peu importe, 'fin comment dire, c'est pas peu importe... mais on leur dit, "acceptez quand même quelque chose, acceptez quand même une formation, même si c'est pas tout à fait votre premier voeu, (hm) ni deuxième, ni troisième, ni des fois aucun des trois ( $\mathrm{hm}$ ), essayez quand même de commencer une formation puisque c'est une formation... parce que vous êtes pas dans la rue, (ouais) parce que vous décrochez pas, parce que voilà". Parce que c'est ça aussi les jeunes qu'on voit nulle part et qui, au mois d'septembre, vont pointer, ou même pas, à la Mission locale. " (Entretien, Nathalie, 40 ans, PLP HAS, en SEGPA depuis 5 ans)

4. Le CFA est un établissement de formation professionnelle en alternance (en général trois semaines en entreprise pour une semaine en centre de formation) qui prépare aux différents diplômes professionnels. L'entrée en CFA nécessite la signature, par le futur apprenti et avec une entreprise, d'un contrat d'apprentissage de la durée de la formation souhaitée.

5. Les dispositifs-relais accueillent des collégiens identifiés en situation de "déscolarisation ", temporairement, en groupe réduit, avec un encadrement spécifique. 
Ainsi, les années de $4^{\mathrm{e}}$ et surtout de $3^{\mathrm{e}}$ sont marquées par la préparation de l'orientation : les PLP prennent des temps en atelier pour parler des formations envisagées l'année suivante. En oral blanc du CFG, les élèves sont systématiquement confrontés à une question sur ce qu'ils envisagent comme poursuites d'études ; et certains PLP dispensent un enseignement hebdomadaire qui réunit découverte des métiers et des formations professionnelles.

Trouver une formation induit bien de trouver une formation professionnelle. Des proximités organisationnelles entre les enseignements professionnels en SEGPA et le lycée professionnel existent : même alternance entre stages et ateliers, même nombre d'heures d'atelier en $3^{\text {e }}$ qu'en CAP (12 heures). D'autre part, les PLP en SEGPA sont par définition des enseignants de LP, mais affectés dans cette section particulière. Et, pour certains, les proximités et la connaissance du lycée sont renforcées par leur propre expérience d'enseignement en LP (durant leur année de stagiaire, ou plus longtemps).

Enfin, les injonctions à poursuivre une formation professionnelle après la SEGPA s'accompagnent aussi de projections dans des métiers, ou plus globalement dans l'emploi. Ces projections prennent la forme de légers renvois à un futur relativement proche "Vous verrez quand vous travaillerez", ou de questions plus directes telles que "Tu veux être quoi plus tard?". Mais plus systématiquement, les visites qu'effectuent les enseignants pour chaque stage réalisé par un élève donnent lieu à questionner une forme de "projection dans le métier" :

"[la visite de stage] c'est une forme d'évaluation plutôt globale, [...] et aussi la projection dans le métier, c'est-à-dire est-ce que euh, on demande au mait' de stage, "est-ce que vous le voyez dans ce métier-là ?" (ah ouais) "Est-ce que vous pensez que ce, ce projet est adapté pour lui ? Est-ce que vous pensez qu'il a des aptitudes pour faire ce métier, etc. ?". Moi j'aime bien que les jeunes entendent ça. » (Entretien, Nathalie, 40 ans, PLP HAS, en SEGPA depuis 5 ans)

C'est en partie au regard de ces projections précoces (elles commencent avant l'année de $3^{\mathrm{e}}$ ) dans des formations professionnelles et dans l'emploi que ces enseignements en SEGPA peuvent être dits professionnels. Cependant, au sein des filières professionnelles diplômantes existantes, les élèves de SEGPA sont incités à envisager certains diplômes plutôt que d'autres.

\subsection{Le CAP comme référence}

Si l'injonction à poursuivre dans une formation professionnelle diplômante est forte, elle ne se fait pas en direction de n'importe quelle formation. L'" espace de projection privilégié, voire exclusif» (Orange, 2010, p. 33) en SEGPA est celui du CAP. Les agents travaillent ainsi à la "délimitation d'un horizon des possibles scolaires " selon le type de diplôme (ibid., p. 39). 
Longtemps, les guides de préparation à l'orientation distribués aux élèves de SEGPA en $3^{\text {e }}$ 'indiquaient, dans leur contenu, que des informations relatives aux $\mathrm{CAP}^{6}$. Les agents eux-mêmes répètent de diverses manières aux élèves ou à leurs parents que le CAP est le premier, sinon l'unique, diplôme à viser. "Leur orientation c'est CAP, c'est uniquement $C A P$ » répète la conseillère d'orientation psychologue (COP) en entretien. Pour Thibaut (53 ans, PLP habitat, en SEGPA depuis 5 ans), il a "fait (s)on boulot " quand les élèves "passent un CAP ». On retrouve, en filigrane, la nécessité actuelle d'avoir un diplôme (Millet \& Moreau, 2011). Pour autant, l'objectif de diplomation n'est pas l'objectif des « 80 \% au Bac » pour ces élèves, mais celui du plus petit diplôme professionnel.

Les agents travaillent en ce sens à " rabattre (les) aspirations" des élèves (Millet \& Thin, op. cit., p. 40) en écartant les métiers pour lesquels il n'est pas possible de commencer par un CAP (médecin, footballeur, psychologue, hôtesse de l'air ${ }^{7}$ ). Les élèves jugés les plus «scolaires " peuvent parfois viser un baccalauréat professionnel. Mais cela leur est souvent déconseillé ; "La marche est trop haute » estime ainsi Christophe en entretien (48 ans, PLP habitat, en SEGPA depuis 17 ans).

Le CAP n'est pas seulement un cap visé en termes d'orientation, mais aussi une ressource dans la construction des enseignements. En l'absence de programme et de référentiel spécifique pour les enseignements professionnels en SEGPA, les PLP s'inspirent des référentiels de CAP et les prennent pour modèle :

"On a la liberté de l'enseignement [...] on tire vers des choses du CAP, voilà (d'accord), mais avec une grande flexibilité en fonction de notre public. [...] ben on doit prendre des éléments du CAP [...], voilà, le référentiel, c'est s'approcher un p'tit peu des choses qu'on demande au CAP. » (Entretien, Christophe, 48 ans, PLP habitat, en SEGPA depuis 17 ans)

Comme ce modèle n'est pas une contrainte officielle, les PLP en SEGPA s'en inspirent, sans toutefois le transposer tel quel. Ainsi, Christophe " tire » les élèves vers le CAP, sans systématiquement confronter ses élèves à des connaissances liées à ce niveau de diplôme. Véronique (65 ans, PLP HAS à la retraite, en SES et SEGPA durant 41 ans), quant à elle, déclare renverser l'ordonnancement des apprentissages du programme de CAP : commencer par la pâte à choux plutôt que par la découpe de légumes. Dans tous les cas, la référence au CAP reste prégnante.

Par ailleurs, en SEGPA, pendant longtemps a existé un enseignement directement issu des programmes de CAP : la "vie sociale et professionnelle", puis la "prévention santé environnement ». Disparu depuis 2015, on en trouve encore des traces, comme l'enseignement de la prévention des risques liés à l'activité physique.

6. Site Onisep.fr, "Collège. L'enseignement adapté : Segpa, Erea », consulté en mai 2015.

7. Ces exemples sont issus de souhaits de métiers exprimés par des élèves ces dernières années à une enseignante (Nathalie, 40 ans, PLP HAS, en SEGPA depuis 5 ans). 
Les enseignants en viennent à penser certains apprentissages comme des formes "d'avances" prises par leurs élèves en vue du CAP. Ils évoquent ainsi tour à tour des «compétences transversales ", pratiques manuelles ou "prérequis ». Autrement dit, en SEGPA, il s'agit d'acquérir " de bonnes bases pour pouvoir commencer un CAP " (Véronique, 65 ans, PLP HAS à la retraite, en SES et SEGPA durant 41 ans).

Si le CAP, plus petit diplôme professionnel, est le seul horizon possible pour les élèves de SEGPA, son accès ne leur est pas garanti, comme son obtention ${ }^{8}$. Les enseignants travaillent, d'une part, à réduire les aspirations des élèves à ce niveau de diplôme et, d'autre part, à les «tirer " vers ce diplôme, à les y préparer au mieux en anticipant, dès la classe de $4^{\mathrm{e}}$, certains apprentissages. La référence au CAP ne vaut néanmoins pas pour n’importe quel CAP, ni lieu de formation.

\section{3 « Commencer par un p'tit CAP» et privilégier un espace « plus protégé »}

Certaines spécialités de CAP sont écartées ou systématiquement déconseillées aux élèves de SEGPA, au nom d'un appel à "être réaliste " ${ }^{9}$. Les élèves sont ainsi invités à éviter la sélectivité de certains CAP, tout comme celle de l'apprentissage (Moreau, 2015 ; Arrighi \& Gasquet, 2010) sous couvert, dans les discours enseignants, d'une certaine "fragilité »" qui nécessite d'envisager des espaces "plus protégés".

Le travail des aspirations, par l'institution, opère tout au long des années de $4^{\mathrm{e}}$ et $3^{\mathrm{e}}$. Il prend la forme d'un "report de la réalisation des aspirations initiales " (Zunigo, 2008, p. 128). La réponse n'est pas que la perspective est inenvisageable, mais qu'elle n'est pas possible directement au sortir d'une $3^{\text {e }}$ SEGPA. Véronique explique ainsi que face aux souhaits désajustés des élèves, elle répond : "Tu peux pas faire c’que tu veux, mais peut-être que tu peux faire quelque chose qui s'en rapproche. " (65 ans, PLP HAS à la retraite, en SES et SEGPA durant 41 ans)

Une des situations emblématiques de ce report des aspirations pour éviter la sélectivité de certaines spécialités est celle de filles qui souhaitent poursuivre en CAP Coiffure ou Petite enfance. Elles en sont progressivement dissuadées. Le premier est caractérisé par une forte attractivité et une plus forte sélection scolaire (Denave \& Renard, 2017) le second connaît un nombre de place "rationné" (Arrighi \& Gasquet, op. cit., p. 108). Parce que le niveau scolaire exigé est trop élevé ou parce que l'offre de places est trop faible face à la demande, les agents conseillent de remettre à plus tard ces vœux et de commencer par

8. Le titre suivant en dit long: "Après leur entrée en sixième en 2007, près de quatre élèves de Segpa sur dix sortent diplômés du système éducatif " (MEN, DEPP, Note d'information $\mathrm{n}^{\circ} 02$, op. cit.).

9. L'appel au réalisme se retrouve dans d'autres filières scolaires ou dispositifs, comme les dispositifs-relais (Millet \& Thin, op. cit.).

10. Cette notion de "fragilité " a été reliée aux notions du XXe siècle d'inadaptation, d'anormalité, voire de handicap socio-culturel, dans un article de Monique Vial \& Marie-Anne Hugon (2009). 
une formation plus polyvalente et plus facile d'accès, comme le CAP Assistant technique en milieux familial et collectif (ATMFC).

Cette invitation à privilégier un CAP plus polyvalent et moins concurrentiel vaut aussi pour les filières masculines :

"Oui si [un élève] est pas prêt, par exemple à aller en... CAP par exemple... ébénisterie ou menuis'rie (hm)... on peut lui conseiller par exemple un CAP MBC [Maintenance des bâtiments de collectivités] où $i$ touche évidemment au bois, mais aussi au carr'lage un peu (hm) bon voilà... » (Entretien, Christophe, 48 ans, PLP habitat, en SEGPA depuis 17 ans)

L'enjeu pour les agents ${ }^{11}$ en SEGPA est bien d'abord de trouver une place à chaque élève dans une formation diplômante plus que de s'assurer qu'ils entrent dans une formation aux débouchés professionnels assurés (Arrighi \& Gasquet, op. cit.). "Commencer par un p'tit CAP » (Véronique, 65 ans, PLP HAS à la retraite, en SES et SEGPA durant 41 ans), c'est finalement mettre un pied dans l'enseignement professionnel et potentiellement pouvoir envisager l'entrée dans une autre formation, un autre CAP, dans un second temps.

Au-delà d'un accent mis sur certains CAP, plutôt polyvalents, avec un plus grand nombre de places et moins attractifs dans une perspective de " gestion des flux " de l'institution scolaire (Briand \& Chapoulie, 1993), les élèves sont également incités à privilégier la voie scolaire plutôt que la voie de l'apprentissage, en raison de sa sélectivité (Moreau, 2015). Ainsi, selon les enseignants, les élèves les plus "autonomes » et les plus "matures", voire les plus "résistants physiquement", peuvent viser un CAP en CFA. En revanche, le lycée est conseillé aux autres en tant qu'espace "plus protégé ", en opposition au "dur» monde du travail. Cette difficulté anticipée renvoie aussi à l'obtention du contrat d'apprentissage - "parce que c'est la crise " dira-t-on en réunion avec les parents d'élèves - et à sa pérennité - "J'en ai vu se faire virer au bout d'un retard» explique la COP (JT, réunion avec les parents d'élèves de 3e, 13.12.2012).

De plus, l'accès au CFA échappe aux procédures scolaires. Ainsi, dans la constitution des vœux d'orientation en fin de $3^{\mathrm{e}}$, les agents de SEGPA incitent toujours les élèves à indiquer au moins un vœu en lycée pour leur assurer une place dans une formation l'année suivante.

Enfin, les élèves perçus comme les moins «bons", les plus "fragiles " ou "en difficulté" sont plutôt exhortés à s'orienter vers un établissement régional d'enseignement adapté (EREA). Appelé "lycée professionnel adapté ", cet établissement scolaire propose des formations sanctionnées par des CAP polyvalents et peu sélectifs comme, dans le département étudié, les CAP MBC et ATMFC.

11. Les enseignants, le directeur ou la directrice de SEGPA, ainsi que, sur la question de l'orientation, le ou la COP. 
À travers l'encadrement des aspirations, des projections dans l'emploi et des contenus des enseignements professionnels, le programme socialisateur en SEGPA est tout tourné vers la voie professionnelle scolaire et vers des CAP peu sélectifs, peu attractifs, plus polyvalents. Néanmoins, les élèves ne sont pas seulement amenés à devenir de futurs lycéens en CAP, mais déjà aussi à devenir de futurs travailleurs.

\section{Socialiser des collégiens à la subordination et à l'exécution}

Faire sa scolarité en SEGPA va de pair avec l'inculcation d'une certaine position dans la hiérarchie des diplômes, la hiérarchie endogène des métiers de CAP (Moreau, 2008b) et la hiérarchie des voies de formation. Mais le cadre socialisateur des enseignements professionnels conduit également à l'apprentissage d'une place dans la hiérarchie du travail et dans l'ordre social. Il renvoie à certaines caractéristiques de la position de subordination et du travailleur d'exécution. Il soumet les corps des élèves à une différenciation progressive de la figure du collégien pour se rapprocher de la figure du travailleur.

\subsection{Les prémices d'inculcation d'une position de subordination}

S'il est bien question des prémices de l'inculcation d'une position de subordination, c'est que les élèves de SEGPA ne font aucune expérience d'une relation salariale en termes de gratifications. Ils ne sont rétribués ni durant leur stage, ni en chantier, ni au restaurant d'application ${ }^{12}$ au sein du collège. Lorsque le chantier se fait à l'extérieur de l'établissement, c'est le collège, et non les élèves, qui reçoit le paiement selon un forfait à l'heure (environ $8 €$ ), établi avec le conseil d'administration de l'établissement, et qui ne prend pas en compte le nombre d'individus au travail (l'enseignant et un groupe d'élèves). L'enseignant qui mène le chantier évalue le nombre d'heures de travail requis et les matériaux nécessaires à sa réalisation sont à la charge du client.

Pour autant, les élèves sont soumis à l'expérience de rapports hiérarchisés. Le respect de l'autorité du patron et celui des horaires sont des exigences régulièrement répétées. Elles prennent la forme indirecte d'une invocation à transposer la situation scolaire en une situation supposée équivalente dans une entreprise. Ainsi, au retour de la pause méridienne, une enseignante invoque un parallèle entre elle et un "patron " pour contrer les plaintes des élèves et réguler leur bavardage.

12. Le restaurant d'application permet aux élèves une mise en situation du travail de restauration au sein de leur établissement. Ils occupent les différents postes de cuisine et de service et servent un repas à des personnes volontaires, contre un paiement qui va au budget de l'établissement. 
Ainsi, en entrant dans l'atelier en début d'après-midi, plusieurs élèves de $3^{\mathrm{e}}$ se plaignent : "On est fatigué "; ils ont déjà passé la matinée en atelier cuisine. L'enseignante leur rétorque : "Vous imaginez ça chez un patron?", puis ajoute : "Vous êtes pas fatigués de la langue. » (JT, atelier HAS, 3 $3^{\mathrm{e}}$, 13.12.2013).

Le respect des horaires et celui du cadre de la convention constituent également les premiers éléments contrôlés lors des visites de stage. L'enseignant qui appelle l'entreprise ou se rend sur le lieu de stage demande inévitablement si l'élève est bien venu aux jours et aux horaires prévus. Enfin, les élèves sont amenés à considérer ces exigences comme premières et indispensables au "bon » stagiaire ou "bon " employé.

Après l'exposé de son expérience de stage, les membres du jury posent plusieurs questions à Aziz. L'une des questions posées est "Et vos qualités et vos défauts?". Aziz répond: "Mes qualités : je viens à l'heure et je fais tout ce que le patron me dit. [Mais] des fois, je crois que je suis lent. " (JT, oraux blancs, $3^{\mathrm{e}}$, 20.12.2013)

Ces exigences d'acculturation à une position de subordination sont maintenues par les enseignants, même en cas de contradictions d'ordre matériel, moral, voire légal. Ainsi, les enseignants attendent non seulement que les élèves, quels que soient leurs moyens de déplacement, respectent les horaires imposés par le maître de stage, mais aussi qu'ils apprennent à ne pas répondre à un "patron ", même lorsque celui-ci "parle mal " à son stagiaire ou à son employé. En situation d'oral de stage, plusieurs pratiques illégales pour des stagiaires de moins de 16 ans ont pu être constatées : une élève a servi de l'alcool au bar, une autre a conduit une voiture pour la déplacer sur le parking du garage, un autre a embauché le matin avant six heures. Face à chacune de ces situations, le jury scolaire est pris dans une tension. S'il peut reprocher au départ la pratique à l'élève, il acquiesce en silence ou invoque la "responsabilité $d u$ patron " dès lors que l'élève mentionne l'ordre direct d'un supérieur. De la sorte, les enseignants font primer in fine l'injonction à obéir au maître de stage. Il en va de même pour les règles d'hygiène ou de sécurité qui sont enseignées aux élèves et qui peuvent être remises en cause en situation de travail.

Une élève se fait reprendre sur l'ordre dans lequel elle dit avoir effectué certaines tâches dans la restauration. Elle explique qu' elle devait dresser la table et ensuite passer le balai. Catherine (PLP HAS) lui fait remarquer que, pour des questions d'hygiène, ça n'est pas dans cet ordre qu'il faut exécuter ces deux étapes. L'élève acquiesce, mais précise : "Ils mont dit de faire comme ça, mais j’osais pas dire ". Le jury ne poursuit pas les questions ou les reproches autour de cette situation et passe à un autre sujet (JT, oraux blancs, $3^{\mathrm{e}}$, 20.12.2013).

L'apprentissage de sa place, de sa position dans l'entreprise, est également perceptible dans les rapports de stages préalables à l'oral de stage pour l'examen du CFG. Un des 
objectifs évalués est explicitement "la capacité [...] à se situer dans son environnement social ou professionnel ${ }^{13}$.

Aux côtés de premières inculcations de la position subalterne, les élèves sont soumis à un encadrement fort de leurs pratiques en atelier qui renvoie à la figure du travailleur d'exécution.

\subsection{Encadrement des pratiques et poste d'exécutant}

L'enseignement professionnel en SEGPA est marqué par un encadrement continu des pratiques des élèves. Ces derniers sont amenés à apprendre à " tenir un poste de travail $»^{14}$.

Cet encadrement passe d'abord par l'exigence du respect des consignes explicitées au début des ateliers et répétées par l'enseignant au fur et à mesure des tâches accomplies durant la séance :

L'enseignante introduit la séance de cuisine : "On écoute et après je vous laisse en autonomie. Aujourd'hui, c'est broyé du Poitou... [...] Le broyé, c'est super simple, y'a pas d'erreurs possibles. Donc le matériel, vous savez ce qu'il faut faire avant?". Un élève : "On nettoie le poste, on se relave les mains". L'enseignante "impose que ce soit enfourné avant la récrée " et de "mélanger à la main". Elle commente et précise la recette à suivre : "Le beurre doit être ramolli, pas fondu. On mélange à la main, donc je veux une hygiène des mains parfaites. "Elle dessine au tableau les traits à faire à la fourchette sur le gâteau et dit : "Vous avez un minuteur, donc c'est vous qui gérez votre cuisson... vous voyez que j'ai durci les choses-là, je n'ai pas écrit le matériel à prendre ". L'enseignante valide le matériel nécessaire suggéré ensuite par les élèves (JT, atelier HAS, 4 ${ }^{\mathrm{e}}, 07.03 .2016$ ).

Les consignes à suivre portent autant sur l'activité de la séance (une recette particulière) que sur la succession des tâches à effectuer (nettoyer le poste, se laver les mains, rassembler le matériel nécessaire) et les manières de les exécuter (mélanger à la main). Mais l'encadrement des pratiques par les consignes est aussi celui des règles d'hygiène et de sécurité à respecter en atelier (ainsi qu'en stage, en principe). Elles recouvrent des tenues particulières (bleu de travail en atelier habitat et ERE ; blouse et charlotte en atelier HAS ; chaussures de sécurité), des gestes et des pratiques spécifiques. Les enseignants attachent de l'importance à la transmission de ces règles. Ainsi, parce qu'elle a oublié sa blouse blanche, une élève est sanctionnée en atelier cuisine. L'enseignante lui impose le port d'une blouse bleue (associée à l'entretien dans cet atelier) et l'élève doit, durant la séance, se charger uniquement de tâches qui n’impliquent pas de toucher les

13. Arrêté du 19 juillet 2016, relatif aux conditions de délivrance du certificat de formation générale, version consolidée au 23 octobre 2019, article 9.

14. Circulaire $n^{\circ}$ IV 67-530 du 27 décembre 1967, «Organisation et fonctionnement des sections d'éducation spécialisée créées dans le cadre des C.E.S. pour l'accueil de déficients intellectuels légers ». 
aliments : nettoyer les ustensiles, préparer la table de pesée, etc. La connaissance de ces règles est également souvent contrôlée lors des oraux de stage.

L'apprentissage à tenir son poste renvoie encore à une socialisation temporelle, d'autant plus observée en atelier cuisine que la transformation des aliments ne peut pas se faire sur plusieurs jours pour des raisons de conservation. Chaque séance est marquée par l'explicitation en amont de la durée de l'activité et par la scansion régulière de rappel de ce temps. Durant la séance de confection de broyés du Poitou, par exemple, l'enseignante qui a indiqué dès le départ l'exigence "d'enfourner avant la récrée ", rappelle à l'ordre les élèves de différentes manières sur la gestion temporelle de l'activité :

La PLP : «Allez on accélère là, y’a la queue [au poste des denrées] et ça me plait pas ». [...]

Ça sonne [1 ${ }^{\text {ère }}$ heure passée], la PLP : «Allez! il reste une heure » [...].

Un élève a fini. L'enseignante lui indique qu'il peut enfourner et doit utiliser le minuteur. La PLP : "Tu peux en faire un autre si tu veux ". L'élève : "Ah bon? j'sais pas si j'vais avoir le temps». (JT, atelier HAS, 4 $4^{\mathrm{e}}$, 07.03.2016)

Ateliers et postes de travail suivent également un ordonnancement spatial que les élèves apprennent à respecter : des espaces spécifiques de rangement pour les outils en habitat, des espaces différenciés selon les tâches de vaisselle ou de transformation des aliments en HAS, des contenants distincts pour le "terreau propre " et le " terreau de récupération" en ERE... Les enseignantes d'HAS enjoignent les élèves à rassembler sur leur plan de travail les ustensiles nécessaires et les ingrédients dans les proportions exigées avant de commencer toute transformation des aliments pour "éviter des allersretours" (Nathalie, 40 ans, PLP HAS, en SEGPA depuis 5 ans).

L'encadrement des pratiques va, pour certaines activités, jusqu'à l'organisation rationnalisée des gestes de travail, alliant respect des règles d'hygiène, de sécurité et d'efficacité temporelle. En entretien, Véronique (65 ans, PLP HAS à la retraite, en SES et SEGPA durant 41 ans) commente une fiche de cours qu'elle a confectionnée. Elle précise l'un des objectifs d'apprentissage consignés : "Travailler de gauche à droite, donc [...] c'est en ergonomie, comme les trois quarts des gens sont droitiers, on prend d'la main gauche, on travaille, on pause à droite."

En atelier, les élèves apprennent que "le temps ne leur appartient pas" (Grignon, 1972, p. 184). De même, l'organisation spatiale de l'atelier et de chaque poste, l'ordonnancement des tâches et les manières de les exécuter, définis par les enseignants, doivent être respectés par les élèves. Malgré les références professorales à "l'autonomie ", les élèves sont socialisés à un poste d'exécutant. 


\subsection{La mise au travail des corps des collégiens}

Lapprentissage de sa place et celui visant à tenir son poste renvoient aussi à des socialisations corporelles. Les corps des élèves sont soumis à des exigences qui participent à les différencier de la figure du collégien et les acheminent vers la figure de l'ouvrier ou de l'employé.

En atelier, les élèves doivent suivre un certain nombre d'exigences corporelles concernant les tenues, les cheveux (attachés), les mains (ongles sans vernis et coupés) et parfois le port de bijoux. Par ce biais, l'encadrement des corps participe à la différenciation et à la spécification de l'élève-travailleur par rapport à l'élève-collégien.

Au sein du collège, la tenue nécessaire à la pratique rythme tous les ateliers par des allersretours aux vestiaires pour l'enfiler ou la retirer. Cette importance de la tenue contribue à marquer sur les corps l'entrée et la sortie de l'atelier et ses activités. Mais elle rend aussi perceptible, aux yeux des autres (collégiens, personnels de l'établissement), les futures positions sociales auxquelles les élèves de SEGPA sont déjà en train d'être préparés.

Stéphane Beaud (1996) analysait les manières dont des élèves de "Bac pro " refusaient ou adaptaient le port du bleu de travail comme indices d'une distanciation de la figure de l'ouvrier, au profit d'une recherche de proximité avec la figure du lycéen. Il évoquait également la gêne des enseignants à imposer le port du bleu de travail.

En SEGPA, les enseignants racontent les difficultés des élèves à se rendre dans la cour de récréation en tenue d'atelier et la plupart d'entre eux se montrent soucieux de ces enjeux dans la gestion des pauses collectives. Marqueur explicite d'une position différente au sein du collège, la tenue d'atelier éloigne de la figure du collégien et rapproche des seules autres personnes portant ce type de tenue dans l'établissement : les agents d'entretien et de la restauration. Les enseignants veillent à éviter à leurs élèves les expériences suscitées par ce marqueur social qu' eux-mêmes n'arborent que peu. Par exemple, ils anticipent la sonnerie de la récréation de dix minutes pour leur permettre de se changer.

Les enseignants contribuent aussi à rendre les élèves attentifs aux attendus vestimentaires des secteurs professionnels fréquentés durant les stages :

Cécile (PE) interroge Mathilde sur la tenue qu'elle devait porter durant son stage en magasin. Mathilde dit d'abord qu'ils s'habillent "normalement». [...] Cécile insiste : "Y'a pas des choses quand même? Tu dis normalement... mais... est-ce que tu peux venir avec un grand décolleté? ". Mathilde : "Ben non, parce que le décolleté, c'est pas fait pour travailler. " (JT, oraux blancs, $3^{\mathrm{e}}$, 16.12.2016)

L'insistance de l'enseignante sur la particularité de la tenue renvoie, de plus, aux exigences de conformité physique plus particulièrement destinées aux filles ou dans les secteurs du service (Denave \& Renard, 2019). Ce rejet du décolleté rappelle l'importance de ne pas "être associée à l'image repoussoir des femmes "vulgaires" "(Lamamra, 2011, p. 341). 
La mise au travail des corps renvoie à d'autres exigences corporelles encore, que sont la dextérité des mains et la "maîtrise du corps ", la valorisation de l'effort et de l'endurance (Grignon, op. cit., p. 183), la douleur et la pénibilité :

"Voilà, et puis on apprend à tenir un couteau, on apprend à travailler, on est à ses mains (hm) les mains c'est... une intelligence ( $\mathrm{hm}$ ) [...] donc euh... avoir d'la mobilité dans les doigts, placer ses doigts, apprendre à se tenir aussi. " (Entretien, Véronique, 65 ans, PLP HAS à la retraite, en SES et SEGPA durant 41 ans)

Ces pratiques corporelles vont avec de premières inculcations d'une certaine persévérance au travail et face à l'effort. En atelier cuisine, en réponse à des plaintes répétées ou à des signes de découragement, de fatigue, voire de douleur, l'enseignante exhorte les élèves à développer une forme d'endurance (Denave \& Renard, 2019) et à passer sous silence ou "banaliser la pénibilité physique» (Lamamra, op. cit., p. 341):

Lucia: "Ça fait mal au bras (à propos d'une pâte plutôt liquide qui nécessite d'être remuée énergiquement) », Catherine (PLP) : "Un pâtissier a toujours les bras bien musclés». Gwenaëlle : "Oh! j'en peux plus », Catherine : "Allez, allez, on y croit! on y croit!». Quelqu'un fait remarquer que Florian a obtenu de la mousse sur le dessus de sa pâte. Catherine : "C'est qu'il a plus de muscles. " (JT, atelier HAS, 3 $3^{\mathrm{e}}, 13.12 .2013$ )

Des discours et des enseignements s'attachent à prévenir les élèves des risques liés aux activités physiques, à l'instar de cette enseignante qui reprend une élève sur sa posture lorsqu' elle règle une table à repasser, pour qu'elle fasse attention à son dos. "Tu vas être une mamie avant l'heure-là ! ", ajoute-t-elle. Pour autant, ces expériences de la douleur et de la pénibilité des tâches sont aussi considérées comme inhérentes au travail. En ce sens, Christophe (48 ans, PLP habitat, en SEGPA depuis 17 ans) explique, lors d'un échange informel en atelier, qu'il a une maladie du canal carpien, celle "de ceux qui travaillent avec leurs mains». En atelier, face à de légers accidents (un élève qui se pique avec une aiguille à coudre, un outil qui dérape sur la main d'un élève sans provoquer de blessure), plusieurs enseignants rétorquent aux élèves : "C'est l'métier qui rentre."

Bien que limitée par l'âge des élèves (moins de 16 ans) et leur statut en entreprise (stagiaire), la socialisation professionnelle en SEGPA est bien effective. Elle est marquée par la subordination et l'exécution. Les élèves sont ainsi amenés progressivement à apprendre à tenir une place subalterne au sein de la structure qui les accueille, à apprendre les règles et les normes professionnelles des tâches effectuées, les contraintes temporelles et spatiales d'un travail d'exécution, l'endurance corporelle et la pénibilité physique de certains travaux. Même s'ils se produisent en école, ces apprentissages sont bien professionnels et contribuent à différencier les élèves de SEGPA des autres collégiens, voire à les distancier de la figure du collégien. 


\section{Une socialisation marquée par la figure de l'ouvrier et de l'employé polyvalent}

Les enseignements professionnels en SEGPA sont présentés dans les discours enseignants et par les dénominations institutionnelles comme non spécialisés, comme ne formant pas à un métier.

Progressivement, la dénomination consacrée pour désigner l'enseignement professionnel en SEGPA est devenue "découverte professionnelle " ${ }^{15}$ et les ateliers spécialisés ont été regroupés en "champs professionnels ${ }^{16}$ (habitat, HAS, ERE). Ces expressions soutiennent l'absence de spécialisation professionnelle. Les enseignants rencontrés parlent parfois de "pré-formation", de "pré-professionnel " ou "simili professionnel ", mais insistent tous sur l'absence d'apprentissage d'un métier et d'une spécialisation. Ceux-ci sont reportés à plus tard, quand les élèves seront dans des formations diplômantes.

Dans les discours enseignants,il s'agit plutôt d'acquérir une "posture professionnelle ", des compétences au travail réputées "transversales" ainsi que de découvrir plusieurs métiers. En ce sens, les élèves sont incités à varier les domaines de stages, à ne pas se focaliser sur un seul projet de métier. Aucun lien n'est de fait exigé entre le domaine professionnel de l'atelier fréquenté et les stages, ou le projet de formation envisagé. D'ailleurs, lorsque les lieux de stage s'écartent des attendus professoraux (en pharmacie, en agence immobilière), les enseignants ne les contestent pas vraiment et mettent en avant l'intérêt d'une expérience dans le "monde professionnel ", "presque peu importe ce stage " (Nathalie, 40 ans, PLP HAS, en SEGPA depuis 5 ans).

Ces déclarations laissent penser que les enseignements professionnels en SEGPA sont presque vides de savoirs et savoir-faire professionnels et ne participent pas à définir, dans leurs contenus, des destins sociaux spécifiques et situés. Or, les élèves de SEGPA sont bien mis en présence d'apprentissages liés à des métiers particuliers dont la spécificité est la grande diversité. Cette variété, associée à l'encadrement des aspirations et à l'injonction aux " p'tits $C A P$ », renvoie au monde des travailleurs polyvalents.

\subsection{Des savoirs et savoir-faire liés à une variété de métiers}

En SEGPA, les enseignements professionnels sont constitués d'un ensemble de savoirs et savoir-faire professionnels associés à une grande variété de métiers.

Les ateliers permettent aux élèves de découvrir, par l'expérimentation, diverses tâches et activités. Plus précisément, les savoirs enseignés recoupent l'apprentissage, sous forme scolaire, des outils et des procédés liés à des métiers. Ainsi, en atelier habitat, lors d'une

15. Circulaire 2015, op .cit. ; Arrêté du 21 octobre 2015, "Classes des SEGPA ».

16. Changement progressif depuis 1989. Première apparition de l'expression dans la Circulaire d'orientation n 89-036 du 6 février 1989, "Enseignements généraux et professionnels adaptés ». 
activité autour du métier de peintre, les élèves apprennent ce que sont et ce qui différencie un "rouleau ", une "patte de lapin" et une "brosse à épousseter ». En atelier HAS, lors d'une évaluation de vocabulaire culinaire, les élèves doivent associer une définition (parmi un ensemble de définitions proposées) à l'un des termes suivants : "émincer", "faire une fontaine ", " abaisser ", " chemiser ", " dorer ", " assaisonner ", " bain-marie ", "saupoudrer», "appareil». Aux côtés de tout un ensemble de termes techniques, les PLP enseignent aux élèves les différents outils ou ustensiles existants et utilisés pour réaliser telle ou telle tâche :

Pour racler le beurre dans le bol [qui a été ramolli au micro-onde] l'enseignante conseille d'utiliser "une maryse "; un élève : "Une quoi ? ", la PLP : "J'ai déjà expliqué! C'est une sorte de spatule. " (JT, atelier HAS, 4 ${ }^{\mathrm{e}}$, 07.03.2016)

L'apprentissage porte ainsi tant sur la dénomination des outils que sur leur utilisation, et notamment sur la pertinence du choix de l'outil selon la tâche à effectuer. Maîtriser cette correspondance fait figure de qualité du professionnel, selon Thibaut (53 ans, PLP habitat, en SEGPA depuis cinq ans). À l'occasion d'une erreur d'un élève, il commente en ce sens qu' " un mauvais professionnel a toujours les mauvais outils".

L'atelier est aussi l'occasion, pour les élèves, d'un "travail sur la matière ou les choses " (Tanguy, 2013, p. 28). Ainsi, en habitat, ils sont amenés à poncer, puis peindre un mur, ou en HAS à réaliser un biscuit roulé et une pâte aromatisée pour fabriquer une bûche de Noël. Encadrés par les PLP qui réajustent ce qu'ils font ou les conseillent, les élèves se voient transmettre, en atelier, des gestes et des techniques liés à l'exercice d'une diversité de métiers. Ainsi, lors d'une séance d'atelier habitat, un groupe d'élèves s'exerce à la taille d'une pierre (ils effectuent une rosace en bas-relief) quand trois autres montent un mur en parpaing. Une charpente, posée sur le sol, témoigne de l'activité des semaines précédentes. Ces savoir-faire professionnels sont également transmis à l'occasion des stages : une élève de $3^{\text {e }}$, lors d'un oral blanc, raconte qu'elle a appris plusieurs gestes et techniques de mécanique automobile en imitant, sur un des côtés de la voiture, ce qu'un professionnel effectuait sur l'autre.

Par ces travaux, et les cours associés, les élèves apprennent des procédés : comment faire un semi, comment planter un pommier d'amour, comment poser des bandes, puis peindre un mur, comment poser du carrelage, comment plier une serviette en trois, comment passer la serpillère, comment repasser un tee-shirt... Et les enseignants leur enseignent les «bons" gestes et les «bonnes» techniques durant les différentes étapes de ces procédés.

En pointant un élève, Christophe (PLP) commente : "Il découvre et il a des bons gestes" en taille de pierre. L'enseignant explique que l'élève a de bons réflexes au bon moment, par exemple quand il arrive à une arrête de la lettre $T$, il sent qu'il faut changer son geste de sens. Il montre à l'enquêtrice la variation à faire avec le ciseau à pierre et le maillet lorsqu' on aborde un angle (JT, atelier habitat, $\left.4^{\mathrm{e}}, 10.05 .2016\right)$. 
Cette variété des métiers, liée aux savoirs et savoir-faire professionnels expérimentés par les élèves, tient aussi aux différentes situations d'apprentissage qu'ils rencontrent. Les élèves font souvent l'expérience de deux ateliers de champs professionnels différents (le principe est généralement d'alterner entre les deux ateliers en $4^{\mathrm{e}}$ et de n'en suivre plus qu'un seul en $3^{\mathrm{e}}$ ).

Mais les stages sont également divers. Les oraux portant sur les stages en font état. Il y est ainsi question d'entretien de locaux, d'accueil et de gestion de clientèle, de démontage de voiture, de réparation d'ordinateur, de préparation de tiramisu au Nutella, de mise en rayon de produits... À l'occasion des repas au sein du restaurant pédagogique de l'établissement, les PLP HAS font tourner les élèves sur différents postes, de l'accueil dans la salle jusqu'au service du café : transformation des aliments, dressage des tables, service des clients (bien souvent des agents de l'établissement scolaire). Quant aux PLP habitat, les chantiers au sein du collège sont aussi marqués par une variété d'activité professionnelle : ils refont les peintures des salles de classe, l'isolation du logement de fonction, le carrelage d'une partie de l'administration, construisent un espace sanitaire dans leur atelier pour leur propre usage, etc.

Savoirs et savoir-faire professionnels, travail sur la matière constituent l'enseignement professionnel en SEGPA. Pour autant, il ne s'agit pas, en effet, d'une socialisation à un métier. Est-ce à dire qu'aucun modèle d'emploi ou de métier ne ressort plus particulièrement de ces enseignements?

\subsection{Polyvalence et « $p^{\prime}$ tit » métier}

Penser les enseignements professionnels uniquement comme non spécialisés occulte la présence de savoirs et savoir-faire liés à des métiers et cantonne la représentation de ce qui est appris en SEGPA à une représentation en creux. En favorisant une appréhension en plein des apprentissages, la diversité des savoirs et savoir-faire enseignés prend sens. Cette focale permet de saisir que le programme socialisateur en SEGPA dirige tout de même les élèves vers certains métiers, ou certains emplois caractérisés par la polyvalence et la faible qualification.

Gabrielle Balazs \& Jean-Pierre Faguer écrivaient, à propos des emplois occupés par des jeunes "non-qualifiés": "Ce qui est commun à tous les emplois, c'est la diversité des tâches" (1979, p. 50). En SEGPA, ce qui est commun aux socialisations professionnelles à l'œuvre, c'est la diversité des savoirs et savoir-faire enseignés. Cette diversité est appréciée comme telle par l'impossibilité d'associer l'ensemble de ces savoirs et savoir-faire à un seul métier spécialisé. C'est cette diversité qui amène à qualifier la socialisation professionnelle en SEGPA de polyvalente. Parler de polyvalence dans ce cas diffère d'une polyvalence associée à des emplois plus qualifiés et aux filières qui y préparent, à l'instar du baccalauréat professionnel, lors de sa création (Maillard, 2017). Elle signifie plutôt une moindre 
spécialisation des enseignements. Elle renvoie à l'idée de "jeune à tout faire " (Balazs \& Faguer, op. cit.) ou encore à la polyvalence de l'ouvrier spécialisé ${ }^{17}$.

L'accent mis par les enseignants sur la "posture professionnelle» et les "compétences transversales " plus que sur la transmission de savoir-faire précis liés à un métier rappelle aussi l'attention portée par " les petits patrons " aux "caractéristiques sociales " des jeunes sans diplôme pour les employer (Ibid.). Le plus important est autant de savoir utiliser ses mains que d'être malléable et docile, adaptable à n'importe quel type de tâche demandée.

La polyvalence des apprentissages rejoint celle des " p'tits $C A P$ » vers lesquels les élèves sont orientés. Les trois CAP les plus présents dans l'encadrement des aspirations des élèves de SEGPA sont caractérisés par une polyvalence des tâches. Le CAP Assistant technique en milieux familial et collectif (ATMFC) peut donner lieu à de l'entretien du linge, des locaux, mais aussi à la préparation de repas. Le CAP Maintenance des bâtiments de collectivités (MBC) implique des activités de prévention, d'entretien et de réparation sur tous les domaines du bâtiment. Le CAP Agent polyvalent de restauration $(A P R)$ - qui comporte explicitement le terme dans son intitulé - renvoie à la diversité du travail dans la restauration : autant du côté du service que de la cuisine (entretien et confection de plats) ou de la gestion des produits. À cette diversité des tâches s'adjoint une position "en bas de l'échelle» dans la division du travail (Avril, 2019). Par exemple, il est précisé, pour le CAP APR, que le titulaire du diplôme, en termes de cuisine, " assemble et met en valeur des mets simples ${ }^{18}$ et pas plus; ce qui renvoie à la position d'un commis de cuisine plus que d'un cuisinier. Les suggestions d'orientation vers ce type de CAP laissent entrevoir comme une sorte de référence minimum à la figure de l'ouvrier ou de l'employé polyvalent.

Comme les métiers visés pour les "petits pauvres " de l'enfance anormale du début du XXe (Muel-Dreyfus, 1975), les destins visés par la socialisation professionnelle en SEGPA renvoient à la polyvalence, à la moindre spécialisation, à un caractère " toucheà-tout » du travailleur. L'enseignement professionnel en SEGPA apparaît alors comme une socialisation à un destin social d'homme ou de femme "à tout faire » (Balazs \& Faguer, op. cit., p. 51).

17. Site Cnrtl.fr, définition "polyvalent, -ente, adj. ", un extrait de l'Encyclopédie pratique de l'éducation en France, 1960, p. 130.

18. Site Onisep.fr, "CAP Assistant Technique en Milieu Familial et Collectif », "CAP Agent Polyvalent de Restauration ", «CAP Maintenance de bâtiments de collectivités ", consulté en janvier 2020. 


\section{Conclusion}

Institutionnellement, la SEGPA n'est pas rattachée aux formations professionnelles. Elle n'est pas sanctionnée par un diplôme professionnel et se situe au niveau du collège.

Cependant, la continuité dans les parcours des élèves entre une scolarité en SEGPA et l'enseignement professionnel a déjà été démontrée (Palheta, 2011). Pour autant, ces liens ne se limitent pas au processus d'orientation ou de division opérées au niveau du collège (ibid.), mais s'étendent aux enseignements qui y sont dispensés. De plus, la SEGPA revêt certaines spécificités par rapport aux autres "classes atypiques " (ibid.) et notamment la durée de scolarité des élèves en son sein (quatre ans pour la majorité) ${ }^{19}$ qui invite à s'intéresser à la socialisation qu'elle encadre. L'étude du programme socialisateur de la SEGPA - c'est-à-dire ce à quoi sont socialisés les élèves - permet de la penser comme une filière professionnelle de formation au sens où elle soumet ses élèves à des socialisations professionnelles.

Les enseignements professionnels en SEGPA constituent une réelle socialisation professionnelle. Les élèves sont amenés à devenir de futurs lycéens de "p’tits " CAP faciles d'accès et polyvalents, mais aussi de futurs travailleurs d'exécution, "touche-à-tout ". Fabienne Maillard (1993) finissait par qualifier certains CAP, en raison de leurs faibles débouchés, leur facilité d'accès, leur polyvalence dans les contenus, etc., comme des sortes de formations intermédiaires entre le collège et le lycée professionnel.

La SEGPA s'inscrit complètement dans une analyse hiérarchisée des espaces scolaires. Pour autant, elle apparaît plutôt à côté du collège (général). De plus, l'analyse défendue se démarque de l'idée de la SEGPA comme une "pré-professionnalisation » définie, notamment, par l'absence de diplôme professionnel en fin de scolarité et le fait qu'elle soit associée davantage au collège qu'au lycée. Au regard de la socialisation à l'œuvre, la SEGPA gagne à être située dans l'espace même des formations professionnelles : celles des " petits » travailleurs d'exécution polyvalents, dont un exemple est l'agent de collectivité, homme ou femme.

Si le programme socialisateur de la SEGPA a suivi les évolutions du système scolaire en s'alignant sur la division collège-lycée, en repoussant l'orientation à la fin de la $3^{\text {e }}$, en incitant les élèves à poursuivre leur formation après 16 ans et à obtenir un diplôme, il reste orienté vers des postes qui, avant ces transformations, étaient accessibles aux jeunes sans diplôme : manœuvre, ouvrier spécialisé, aide jardinier, aide en cuisine, domestique, etc.

Enfin, ces analyses participent à préciser la relativité de l'unicité du collège en examinant (en plein) ce qui constitue et différencie la SEGPA de la filière générale au

19. En termes de durée de scolarisation, seules des filières du handicap comme l'ULIS (Unité localisée pour l'inclusion scolaire) peuvent accueillir aussi longtemps des élèves, mais elles sont absentes du Panel 1995, et ne sont pas analysées par Ugo Palheta. 
collège (mais également d'autres scolarités dérogatoires au collège unique) ${ }^{20}$. Les élèves en SEGPA sont conduits plus précocement que les autres à ne plus être seulement collégiens, mais déjà à s'envisager en CAP et travailleurs.

Ainsi, les socialisations professionnelles initiales ne commencent pas nécessairement à l'entrée dans une formation professionnelle diplômante. Elles peuvent lui être antérieures et s'opérer dans un contexte scolaire. Les élèves, selon les modalités de leur scolarisation, peuvent avoir déjà incorporé certains rappels à l'ordre social.

Bien sûr, ces analyses sont celles du cadre socialisateur de la SEGPA. En ce sens, elles invitent à questionner, à l'occasion d'autres recherches, la transmission et la réception des dispositions ici dégagées. Il est possible de formuler l'hypothèse que ces socialisations ont des effets, au moins à court terme, sur les orientations des élèves. L'étude des inscriptions une année après la SEGPA, sur deux années différentes, dans le département d'enquête, conforte cette hypothèse. En effet, parmi les sortants de $3^{\text {e }}$ SEGPA en 2011, 140 jeunes sur 154 sont inscrits en CAP en LP l'année suivante et $35 \%$ sont dans l'un de ces trois CAP : ATMFC (principalement), MBC et APR. En 2014, une même prédominance des CAP ATMFC et MBC est constatée : ensemble, ils représentent $34 \%$ des inscriptions pour les élèves sortant de $3^{\text {e }}$ SEGPA quand chaque autre CAP ne représente pas plus de $4,5 \%$ des inscrits.

D'autre part, cette socialisation professionnelle connait a priori des variations pour les élèves, selon la SEGPA fréquentée, l'enseignant d'atelier, ou les stages effectués. Ils peuvent alors être plus ou moins amenés à consolider certaines dispositions (comme par exemple le rapport au temps plus particulier en atelier cuisine). Cette socialisation est aussi un début de socialisation professionnelle puisque, selon la suite des parcours, l'inculcation de ces différentes dispositions connaîtra des renforcements ou des transformations variables.

Reste que, et malgré certains discours officiels ou ceux d'agents de l'institution scolaire, les élèves sont bien socialisés de manière particulière par leur passage en SEGPA, et notamment sur l'aspect professionnel de leur scolarité. Ils sont façonnés à devenir des élèves de LP dans des CAP qui préparent à des métiers polyvalents ; ils sont mis au travail et confrontés à certaines caractéristiques des postes subalternes et d'exécution ; ils commencent à apprendre dans leur corps ce que nécessite la tenue d'un poste de travail ; ils acquièrent des savoirs et savoir-faire professionnels et travaillent déjà de leurs mains la matière, et ce, dès quatorze ans.

20. En 2016, sur un total qui dépasse 3200000 élèves en collège, moins de 1000 élèves sont scolarisés en "classes relais et autres dispositifs" et autour de 31000 élèves sont en ULIS. Par ailleurs, le nombre d'élèves en SEGPA dépasse les 86000 (DEPP-RERS, op. cit., p. 87). Un certain nombre de filières dérogatoires se concentrent sur la fin du collège. En ce sens, d'après l'étude du Panel 1995 d'Ugo Palheta (2012), les élèves en «classes atypiques» (dont la SEGPA) représentent 11,1\% des effectifs d'une cohorte d'élèves entrés en $6 e$ en 1995, trois ans après celle-ci. 


\section{Bibliographie}

Avril C. (2019), "Les emplois du bas de l'échelle ne sont pas qualifiés », in Masclet O., Misset S. \& Poullaouec T., La France d'en bas ?, Paris, Le Cavalier Bleu, pp. 37-42.

Avril C., Cartier M. \& Serre D. (2010), Enquêter sur le travail, Paris, La Découverte, 286 p.

Arrighi J.-J. \& Gasquet C. (2010), «Orientation et affectation : la sélection dans l'enseignement professionnel du second degré », Formation Emploi, n 109, pp. 99-112.

Balazs G. \& Faguer J.-P. (1979), «Jeunes à tout faire et petit patronat en déclin », Actes de la recherche en sciences sociales, vol. 26-27, pp. 49-55.

Beaud S. (1996), "Les "bacs pro" ", Actes de la recherche en sciences sociales, vol. 114, pp. 21-29.

Bertrand J. (2012), La fabrique des footballeurs, Paris, La Dispute, 165 p.

Briand J.-P. \& Chapoulie J.-M. (1993), "L'institution scolaire et la scolarisation : une perspective d'ensemble ", Revue française de sociologie, $\mathrm{n}^{\circ}$ 34, pp. 3-42.

Darmon M. (2015), Classes préparatoires, Paris, La Découverte, 328 p.

Denave S. \& Renard F. (2015), «Aspirants mécaniciens, aspirants coiffeurs », Terrain et Travaux, vol. 2, n' 27 , pp. 59-77.

Denave S. \& Renard F. (2017), «L'orientation en CAP métiers de l'automobile et coiffure ", Education et formation, n ${ }^{\circ}$ 93, pp. 43-66.

Denave S. \& Renard F. (2019), "Des corps en apprentissage », Nouvelles questions féministes, vol. $38, \mathrm{n}^{\circ} 2$, pp. 68-84.

Grignon C. (1972), L'ordre des choses, Paris, Les Éditions de minuit, 367 p.

Lahire B. (1993), La raison des plus faibles, Lille, Presses universitaires de Lille, 188 p.

Lamamra N. (2011), "La formation professionnelle en alternance, un lieu de transmission des normes de genre? ", Revue de psychologie du travail et des organisations, vol. 17, $\mathrm{n}^{\circ} 4$, pp. 330-345.

Maillard F. (1993), " De la professionnalisation à la remédiation scolaire : les multiples fonctions d'un diplôme ", Formation Emploi, n 42, pp. 25-32.

Maillard F. (2013), "Les "petits" diplômes professionnels français dans la politique éducative et sur le marché du travail ", Cahiers de la recherche sur l'éducation et les savoirs, $\mathrm{n}^{\circ} 4$, pp. 167-187.

Maillard F. (2017), «Le baccalauréat professionnel de 1985 à nos jours : d'une singularité à l'autre ", Revue Française de Pédagogie, vol. 1, n 198, pp. 11-22. 
Millet M. \& Thin D. (2003), "Une déscolarisation encadrée ", Actes de la recherche en sciences sociales, vol. 4, $\mathrm{n}^{\circ} 149$, pp. 32-41.

Millet M. \& Moreau G. (dir.) (2011), La société des diplômes, Paris, La Dispute, «Etats des lieux ", $280 \mathrm{p}$.

Moreau G. (2008a), «Apprentissage : une singulière métamorphose », Formation Emploi, $\mathrm{n}^{\circ} 101$, pp. 121-133.

Moreau G. (dir.) (2008b), Les apprentis ligériens en 2006/2007, Rapport au conseil régional des Pays de la Loire, $179 \mathrm{p}$.

Moreau G. (2015), «L'apprentissage, un bien public? ", L'orientation scolaire et professionnelle, vol. $44, \mathrm{n}^{\circ} 2$.

Muel-Dreyfus F. (1975), "L'école obligatoire et l'invention de l'enfance anormale ", Actes de la recherche en Sciences Sociales, vol. 1, n 1, pp. 60-74.

Orange S. (2010), "Le choix du BTS ", Actes de la recherche en sciences sociales, vol. 3, $\mathrm{n}^{\circ} 183$, pp. 32-47.

Orange S. (2013), L'autre enseignement supérieur, Paris, PUF, "Éducation et Société ", $226 \mathrm{p}$.

Palheta U. (2012), La domination scolaire, PUF, Paris, 360 p.

Palheta U. (2011), « Le collège divise », Sociologie, vol. 2, nº 4, pp. 363-386.

Tanguy L. (2013), « Apprentissage en entreprise et formation professionnelle en école : une mise en perspective des années 1950 aux années 1990 ", Revue française de pédagogie, $\mathrm{n}^{\circ} 183$, pp. 27-38.

Vial M. \& Hugon M.-A. (2009), «Anormalité, débilité, inadaptation, handicap socioculturel, fragilité : une histoire sans cesse recommencée ? ", Spécificités, vol. 1, n² pp. 21-32.

Zaffran J. (2010), "Entrer en segpa et en sortir ou la question des inégalités transposées », Formation Emploi, ${ }^{\circ} 109$, pp. 85-97.

Zolesio E. (2012), "La chirurgie et sa matrice de socialisation professionnelle ", Sociologie, vol. 3, n 4, pp. 377-394.

Zunigo X. (2008), "L'apprentissage des possibles professionnels ", Sociétés contemporaines, vol. 2, n 70, pp. 115-131. 